\title{
Stigma in Mental Illness: Evidences and Strategies
}

\section{Authors: Elsa Reis', Lígia Castanheira1, Custódio Rodrigues', Tânia Cavaco1, João Rema1, Rodrigo Santos ${ }^{1}$, Maria João Gonçalves ${ }^{1}$ \\ Psychiatry and Mental Health Service - Hospital de Santa Maria, Centro Hospitalar Lisboa Norte, E.P.E.;}

\section{Background and Aims}

The concept of stigma refers to a set of negative attitudes and beliefs about certain subject. In mental health, patients deal with social stigma and self-stigma, which affects treatment adherence and prognosis. Therefore there has been an increased interest in developing new interventions to diminish stigma.

\section{Materials and Methods}

Review of published literature (PubMed, Medline).

\section{Results}

Several articles explored ideas of dangerousness, criminality, and blame of adults with mental illness as well as notions of incompetency, dangerousness and punishment. In general, adults with schizophrenia, depression, alcohol or drug dependence are more likely to be seen as violent to others, compared to a person with 'normal' troubles.

Education was negatively related with dangerousness with higher levels of education associated to perceiving these people as less dangerous. Additionally personal contact with someone with mental illness decreased perceptions of dangerousness. At the same way, beliefs of shame, blame, incompetency and punishment are very common.

Overall, stigma seems relatively stable over time. In general, the American public seems to hold positive opinions in seeking professional help for mental health problems and these attitudes seem to be improving over time. Older age and being female are factors associated with approving mental health treatments. In fact, younger males describe more negative opinions.

It has been found that experiencing discrimination is linked with internalizing the negative perceptions of mental illness (self-stigma). It is consensual that self-stigma has important negative effects meaning a major obstacle to recovery. As a result, there has been investigation about interventions to ameliorate self-stigma. Within this intervention there are several mechanisms engaged, in different forms. Firstly, psychoeducation and information to counter-act myths is common to all approaches. Healthy Self-Concept, for example, was created to be used in people who have experienced their first psychotic episode. NECT (Narrative Enhancement and Cognitive Therapy) and ESS (Ending Self-Stigma) are group-based interventions that can be used with professional facilitators.

There are some positive results, but outcome and implementation research is still in early stages, so future evaluation is necessary to understand the true potential of these programs.

On the other hand, it is important to reflect about how public opinion is made and influenced. Media is a very relevant factor to be considered and should be used to clarify myths, to deliver anti-stigma messages and promote promote helpseeking behaviours.

\section{Conclusions}

Stigma refers to beliefs of dangerousness, shame, blame, incompetency and punishment towards people with mental illness. These ideas varies with culture and other sociodemographic factors. The public stigma results in self stigma, which affects deeply the outcomes and recovery of patients. All of us as society have a part in fight against mental illness stigma, particularly media, by its influence and power.

Various methods have been criated to diminish self-stigma, with particular characteristics. There are some studies with benefic results. However it is necessary further investigation to prove that and to clarify more specifics.

Bibliography: 1- Parcesepe A., Cabassa L., Public Stigma of Mental IIIness in the United States: A Systematic Literature Review, Adm Policy Ment Health . 2013 Septembe ; 40(5): 